Article

\title{
Improvements of Piezo-Actuated Stick-Slip Micro-Drives: Modeling and Driving Waveform
}

\author{
Xuan-Ha Nguyen ${ }^{1, *}$, Tien-Hiep Mau ${ }^{1}$, Ingo Meyer ${ }^{2}$, Bao-Lam Dang ${ }^{1}$ and Hong-Phuc Pham ${ }^{1}$ \\ 1 Department of Machine Design and Robotics, School of Mechanical Engineering, Hanoi University \\ of Science and Technology, Hanoi 112452, Vietnam; hiepmautien@gmail.com (T.-H.M.); \\ lam.dangbao@hust.edu.vn (B.-L.D.); phuc.phamhong@hust.edu.vn (H.-P.P.) \\ 2 Engberts $\mathrm{GmbH}, 26188$ Edewecht, Germany; ingo.meyer@engberts.de \\ * Correspondence: ha.nguyenxuan@hust.edu.vn; Tel.: +84-24-3868-0101
}

Received: 29 December 2017; Accepted: 5 February 2018; Published: 7 February 2018

\begin{abstract}
Modeling and waveform optimization are important research topics for piezo-actuated stick-slip micro-drives. In this paper, the dynamics of piezo-actuated stick-slip micro-drives (PASSMDs) are theoretically investigated. We introduce an extended model taking the dynamics of the piezo actuators into account. The model combines the whole macroscopic movement of the drive's runner and actuators and the microscopic behavior of the frictional contacts in a hybrid dynamic simulation. The macroscopic movements are described via Newtonian mechanics, while the microscopic behavior is computed using the method of dimensionality reduction. Two important characteristics of the drive, the critical actuation amplitude and the force generation, are systematically analyzed. The numerical simulation results show a fine agreement with experimental data of the previously published work. The critical actuation amplitude is found to depend on the behavior of the guiding contacts, the dynamics of the actuators and their interaction. Furthermore, a novel driving waveform, which allows us to increase the operational velocity for the drive, is proposed. The waveform is derived by exploiting micro-vibration and considering the dynamic contact status. Simulation results show that the average velocity of the drive is heightened by about $15 \%$. The performance of the drive is therefore improved.
\end{abstract}

Keywords: contact mechanics; micro-robot; method of dimensionality reduction; piezo actuator; stick-slip micro-drive

\section{Introduction}

For many micro and nano manipulation tasks, the need for small-sized and ultra-high-precision positioning and manipulation devices is essential [1]. Among them, piezo-actuated stick-slip micro-drives (PASSMDs) have received much attention given their main advantages of a very simple design consisting of only few parts (see Figure $1 \mathrm{a}$ ) and noticeable working properties. PASSMDs are guided and driven by piezo actuators supplied by saw-tooth driving voltages (see Figure 1d). The motion of these devices consists of sequences of stick and slip-phases. In the stick phase (see Figure 1b), the mobile part, called the runner, is slowly displaced along with slow expansion of the actuators due to the static friction force in the guiding contacts. This phase is followed by the slip-phase with a rapid contraction of the actuators in the opposite direction (see Figure 1c). The runner cannot fully follow this displacement and thus slides relatively with respect to the actuators. As a result, after a small back-step, a step of the runner is generated. This motion principle has an advantage for movements of the runner with not only very high resolution but also theoretically with unlimited range, although the displacement of the actuators is limited. 


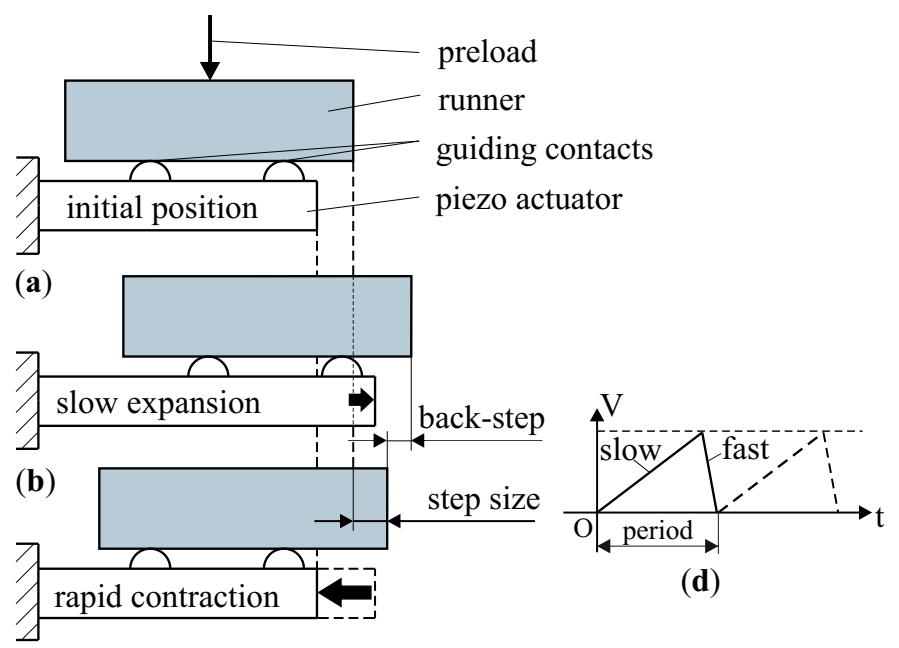

(c)

Figure 1. Components and working principle of piezo-actuated stick-slip micro-drives: (a) initial position; (b) stick-phase; (c) slip-phase; (d) saw-tooth-like driving voltage applied on the piezo.

The modeling and control of PASSMDs are important research topics. However, although numerous prototypes and systems based on the stick-slip principle have been introduced (see [2,3] and chapter 2 of [4]), the proof-of-concept of individual drives is the main focus. There have been only few works concerning the physical modeling of micro-drives intensively [4-13]. All of these works have tried to model frictional behavior in the guiding contacts with or without considering dynamic effects of the piezo actuators.

There is an important phenomenon of PASSMDs experimentally observed. If the actuation amplitude of the piezo actuators is smaller than a critical value, no measurable step of the runner is obtained. This means the runner only oscillates around its initial position without step generation. In this paper, we call the actuation amplitude at this critical value the "critical amplitude, $A_{\mathcal{c}}$ ". Explanations for this phenomenon are classified into two groups:

- The work of van der Wulp [5] is classified into the first group. In this work, the author explained that the critical amplitude is caused by the presliding, a maximal deformation of the guiding contacts before a full slip occurs, and the dynamic effects of the piezo actuators. The guiding contacts are described by the classical tangential contact theory. The piezo actuators are modeled as a mass-spring-damper system. However, only simple analytic formulations for critical amplitude were generated. Dynamic simulations were not implemented. Therefore, further investigation should be performed.

- In the second group [4,7-13], the authors explained the critical amplitude by only the presliding. Dynamic effects of the piezo actuators do not influence this phenomenon. Therefore, the friction model for the guiding contacts was concentrated on.

Breguet used the LuGre model, invented by Canudas de Vit et al. [14], for modeling a linear stick-slip micro-drive [7]. This model is capable of predicting basic friction properties. However, the presliding cannot be covered, as the deformation of the contacts is considered as plastic. The presliding is simply stated as "contact deformation". Peng and Chen [8] exploited the elastoplastic model (EPM) [15], an extension of the LuGre model, for their investigations. Because the EPM takes the elastic property into account, it allows one to predict the presliding, but it is only qualitative. Consequently, in $[9,10]$, Edeler et al. made an adaption for the EPM by adding six empirical parameters to establish the CEIM-model (Christoph Edeler - Ingo Meyer Model). This model shows a higher simulation quality. 
It is known that all of the above-mentioned models are extended from Dahl's single-state friction law [16], which is based on the interaction of surface asperities of contacting bodies. These models are used in such a way that all important parameters are empirically chosen or fitted. The real physical properties of frictional contacts are neglected. Therefore, a new approach was first proposed in [11] and further studied in $[4,12,13]$. In this approach, the authors used the standard tangential contact theory and applied the method of dimensionality reduction (MDR) (see $[17,18]$ ) to describe the friction behavior of the guiding contacts. The model of this approach, namely, the TC-MDR (tangential contact with MDR), permits a much higher modeling quality, as all important influencing parameters of the contacts are taken into account and thus none of the empirical or fitted parameters are used. However, the relation between the critical amplitude and the presliding in the case of the multi-contact model is still qualitatively explained.

While all above-mentioned models allow for an increasing simulation quality, we believe that the explanation for the critical amplitude phenomenon has not been fully satisfied yet, as its relation to the dynamic effects of the piezo actuators is not considered. Therefore, in this work, we extend the TC-MDR model by taking the dynamics of the piezo actuators into account to establish a new model. This model will allow us to explain clearly the causation of the critical amplitude phenomenon. Additionally, the remaining deviation between experiments and simulations can be analyzed and narrowed.

The optimization of the driving waveform is significant for the higher performance of PASSMDs. An optimized waveform may allow us to increase the step size and reduce back-step and micro-vibration, and thus it results in a faster operational velocity, a higher repeatability and less chaos for the drives. There have been both hardware- and model-based approaches. For the hardware-based approach, in [19], Špiller and Hurák introduced a hybrid charge amplifier. The device allows us to provide a high-voltage asymmetric saw-tooth-like signal while maximizing the slew rate and compensating for the hysteretic behavior of the piezo actuator. As a result, it exhibits the minimum back-step and thus induces less micro-vibration. The model-based approach has often been used [4,20-22]. In [20], Bergander and Breguet used a method called "signal shaping" to optimize the driving waveform for a linear stick-slip micro-stage. The method permits reducing vibrations of the piezo actuators efficiently and thus heightens the operational velocity as well as the repeatability. Nevertheless, this method can only be applied for drives having actuators with a relatively low natural frequency. Recently, Hunstig et al. [21,22] proposed a novel driving method for a macroscopic stick-slip drive with the actuators' stroke of $27 \mu \mathrm{m}$. It is known that the optimized signal allows the drive to operate with very high velocities in the slip-slip regime, while the reachable maximum velocity in stick-slip operation is principally limited. Neither the works of Bergander et al. nor Hunstig et al. consider the dynamic behavior of the guiding contacts influencing the drivers' performance. This is particularly important for micro-drives. Consequently, a new driving waveform was first introduced in [23] and was further studied in [4]. On the basis of a simplified model of a micro-drive using the MDR, the motion of the drive in one period is analyzed, in which the dynamic behavior of the guiding contacts is involved. This leads to an optimized waveform allowing us to reduce micro-vibrations as well as the back-step. The experimental validation results show that the performance of the drive is substantially improved with possible driving frequencies up to $13 \mathrm{kHz}$.

None of published works known to the authors exploit the micro-vibration as a source to increase the step size and thus the operational velocity for the drives. In this work, we propose a new ideal for the driving signal. The optimized waveform is obtained by analyzing the movement of the runner and its micro-vibration on the basis of the dynamic model of the guiding contacts using MDR. This new ideal will allow us to significantly increase the step size of the runner and thus the speed.

Consequently, the contribution of this paper is twofold. On the one hand, an extended model is derived to describe the whole macroscopic movement of the drive and the microscopic dynamic behavior of the guiding contacts. A validation of the model indicating its accuracy is provided. On the other hand, a new driving waveform is proposed, which allows us to increase the operational velocity for the drives. 


\section{Investigated Drive}

The drive investigated in this work was first introduced by Edeler [10] and was adapted by the first author of this paper [12]. Experimental results for the characterization of the device are presented in detail in [4]. In order to validate our further improvements for the drive, we use these already published experimental results. For the sake of brevity, in the following, we shortly describe the construction and working principle of the device.

The test stand for the investigated drive, presented in [12] by the first author of this paper, is shown in Figure 2a. The linear drive consists of a runner, which is guided and driven by six ruby hemispheres. These ruby hemispheres are glued onto six piezo actuators. The actuators are arranged on both sides of the runner with an angle of $90^{\circ}$ to each other (two on the right and four on the left; cf. Figure $2 b, c$ ) and are mounted onto the actuator holders. This arrangement allows for horizontally translational forward and backward movements of the runner. The actuator holder on the right is fixed, whereas that on the left is guided by a very low friction linear ball bearing. This will make sure that the preload applied on the runner-hemisphere contacts is only impacted by the preload spring. The level of the preload is measured by a force sensor (M17 by ATI Industrial Automation, Pinnacle Park Apex, NC, USA). The position of the runner is recorded by the high-resolution laser interferometer (SP-120 by SIOS Meßtechnik GmbH, Ilmenau, Germany). Measurements of the forces generated by the drive are implemented with a miniature load cell (M31 by Sensing and Control Automation and Control Solutions Honeywell, Golden Valley, MN, USA).

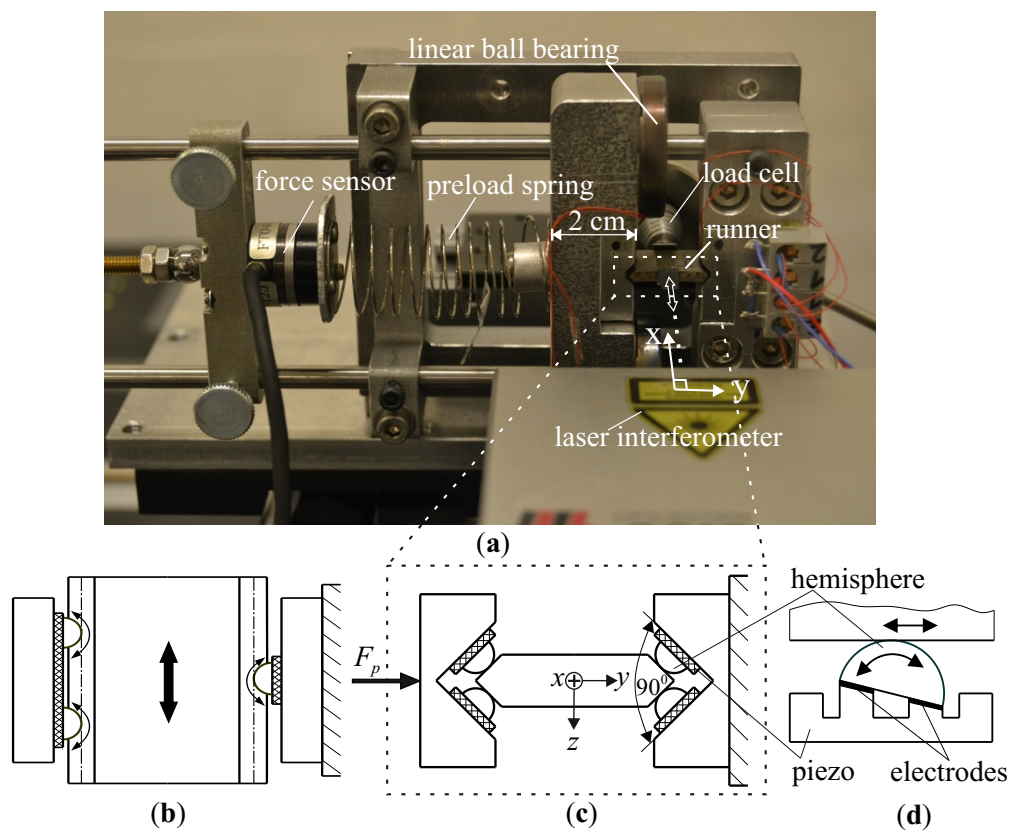

Figure 2. Setup of the experimental test stand: (a) photo of the real setup; (b) CAD-based schematic top-view (CAD-Computer Aided Design); (c) CAD-based schematic front-view; (d) CAD-based schematic view of the piezo actuators [12].

The design of the piezo actuators is schematically shown in Figure $2 \mathrm{~d}$. The ruby hemisphere is glued on two segments of the structured actuator, which is fabricated out of piezoceramic plates (PIC-151 by PI Ceramic GmbH, Lederhose, Germany). Two electrodes are coated on the top surface of the piezo segments. When two saw-tooth-like voltages with opposite signs are supplied on these electrodes, the piezo segments will be deformed, leading to the saw-tooth-like rotation of the ruby hemisphere. The synchronous saw-tooth-like rotation of all six ruby hemispheres results in the translational motion of the runner according to the stick-slip principle. The applied voltages on the piezo have a maximal amplitude of $300 \mathrm{~V}_{p p}$ and a typical slew-rate of $150 \mathrm{~V} / \mu \mathrm{s}$. The maximal 
displacement of the contact center of each hemisphere depends on its radius and the dimension of the piezo segments, as well as the amplitude of the driving voltages. This displacement is denoted as the actuation amplitude. All important influencing parameters, including the radius of the frictional contacts, preloads, and the control amplitude of the driving signal, can be independently controlled and investigated.

\section{Modeling}

In this paper, we extend the model introduced in [11,12] so that the dynamics of the piezo actuators can be investigated. Instead of considering the piezo actuators as ideally rigid, we model them as mass-spring-damper systems. Movements of the runner and the actuators are described by the macroscopic model. The microscopic model represents the interaction between the runner and the hemispheres through contact forces.

\subsection{Macroscopic Modeling}

The macroscopic model to describe overall movements of the investigated drive is shown in Figure $3 \mathrm{a}, \mathrm{b}$ using a free-body diagram. The runner with a mass $m_{r}$ displaces in a horizontal direction. The six actuators are represented by the mass-spring-damper systems with a mass of $m_{a}$, a stiffness of $k_{p}$ and a viscosity of $c_{p}$. We suppose that these parameters for all actuators are identical. Discrepancies due to the fabrication tolerance are neglected. Each mass-spring-damper system drawn in Figure 3a is depicted for two actuators. The normal forces applied on the contacts are calculated from the preload through the geometrical factor and the number of contacts on each side of the runner. It is assumed that the normal force on all contacts of one side of the runner at each level of the preload is identical. Because the actuators are arranged with a $90^{\circ}$ angle to each other (see Figure $3 \mathrm{~b}$ ), the normal forces on the contacts are

$$
F_{N 1}=\frac{F_{p}}{2} \times \frac{1}{\sin \left(45^{\circ}\right)} ; F_{N 2}=\frac{F_{p}}{4} \times \frac{1}{\sin \left(45^{\circ}\right)}
$$

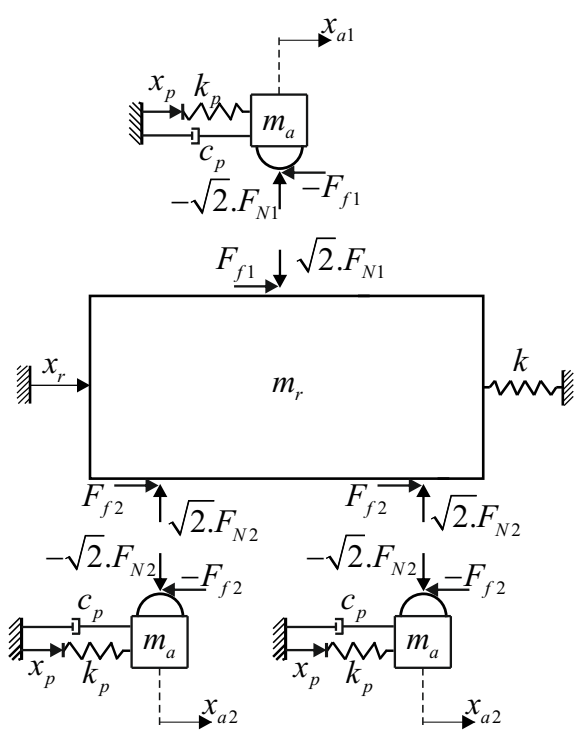

(a)

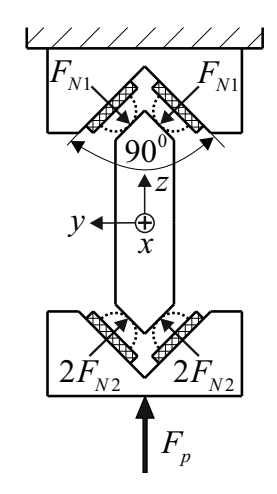

(b)

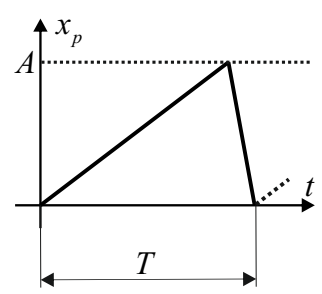

(c)

Figure 3. Macroscopic model for the investigated drive with six actuators: (a) front view with only three of six actuators represented (the factor $\sqrt{2}$ is added in front of $F_{N j}(j=1,2)$ because of their projections in $x z$ plane); (b) side view; (c) saw-tooth-like deformation of the piezo. 
As explained in [12], the influence of the gravity force of the runner is neglected. Additionally, when saw-tooth-like driving voltages are applied on the piezo, as shown in Figure 3c, the translational displacement of the hemispheres is achievable.

The coordinates $x_{r}$ and $x_{a j}(j=1,2)$ describe the displacement of the runner and the actuators on both sides; $x_{p}$ is the desired piezo actuator position induced by the electrical field. $F_{g e n}$ is the generated force applied to the load cell, which is modeled by a linear spring with stiffness $k$. Applying Newton's second law for the movement of the runner and actuators yields

$$
\begin{gathered}
m_{r} \ddot{x}_{r}=-k x_{r}+2 F_{f 1}+4 F_{f 2} \\
m_{a} \ddot{x}_{a 1}=k_{p}\left(x_{p}-x_{a 1}\right)-c_{p} \dot{x}_{a 1}-F_{f 1} \\
m_{a} \ddot{x}_{a 2}=k_{p}\left(x_{p}-x_{a 2}\right)-c_{p} \dot{x}_{a 2}-F_{f 2}
\end{gathered}
$$

where $F_{f 1}$ and $F_{f 2}$ are the friction force between the hemispheres and the runner at an instant of time $t$ computed by the microscopic model. It is noticed that Equation (3) is established using the electromechanical model of the piezo actuators, where the term $k_{p} x_{p}$ is the internal force caused by piezoelectric effects, $k_{p} x_{a j}$ is the elastic force of the piezo, and $c_{p} \dot{x}_{a j}$ is the damping force. For more details, readers are recommended to refer to [4].

\subsection{Microscopic Modeling}

The physical description of the micro-contacts between the runner's surfaces and the ruby hemispheres is described via the microscopic model. In this work, we use the model introduced in [11]. On the basis of this model, the micro-contacts are considered as standard tangential contacts with slip, for which important influencing parameters such as the normal force $F_{N j}$, the radius of the hemispheres $R$ and the material's properties can be modeled. By applying the MDR $[17,18]$, the considered contacts are simplified to an equivalent one-dimensional contact (see Figure 4). In this contact, a rigid sphere is in contact with an elastic foundation having a set of springs. The springs have normal stiffness $\Delta k_{z}$ and tangential stiffness $\Delta k_{x}$, which are chosen according to the following rules:

$$
\Delta k_{z}=E^{*} \Delta x ; \Delta k_{x}=G^{*} \Delta x
$$

where $\Delta x$ is the distance between the springs, and $E^{*}$ and $G^{*}$ are the equivalent elastic and shear modulus. The radius of the one-dimensional sphere $R_{1}$ must be

$$
R_{1}=\frac{R}{2}
$$

For the formal proofs of the exactness of the method, readers are recommended to refer to $[11,12,17,18]$.

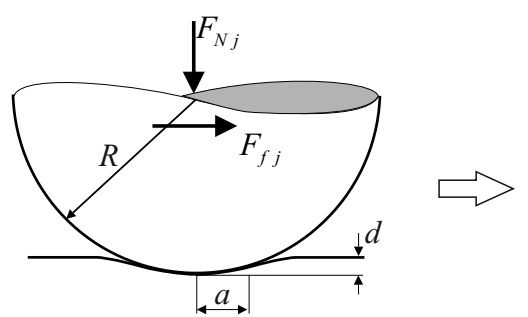

(a)

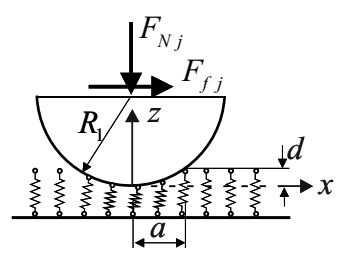

(b)

Figure 4. Microscopic model: (a) three-dimensional Hertzian contact between a hemisphere and the runner; (b) one-dimensional equivalent contact with elastic foundation [11]. 
Using this simplified model, we can predict the dynamically changing contact area and thus compute the friction force. At each instant of time $t$ of a dynamic simulation, the changing stick and slip area of the contact is determined by the state of the springs in contact. Depending on the displacement of the runner $x_{r}$ and the hemispheres $x_{a j}$, all springs within the contact are deflected with an amount $u_{x, i}(t)=x_{r}(t)-x_{a j}(t)$. If $u_{z, i}$ denotes the deflection of a spring $i$ in the normal direction, then the spring forces in the tangential and vertical direction are $f_{x, i}=\Delta k_{x} u_{x, i}$ and $f_{z, i}=\Delta k_{z} u_{z, i}$, respectively. A spring slips if the stick condition is fulfilled: $f_{x, i}=\Delta k_{x} u_{z, i}>\mu f_{z, i}$. Then the spring force becomes $f_{x, i}=\mu f_{z, i}$, and the tangential displacement changes to $u_{x, i}=\frac{\mu f_{z, i}}{\Delta k_{x}}$ accordingly ( $\mu$ is the friction coefficient). As a result, the friction force in each contact $F_{f j}(t)(j=1,2)$ is the summation of all tangential spring forces:

$$
F_{f j}(t)=\sum_{i=1}^{N} f_{x, i}(t)
$$

where $N$ is the total number of springs within the contact.

\section{Validation}

In this section, we present the simulation results for the investigated drive using the model introduced in Section 3. It is shown that the introduced model allows us to explain important phenomena of the drive, including the critical amplitude and the force generation, more clearly and exactly than previously published works. Simulations were performed using a hybrid dynamic algorithm [11,12], which combines the macroscopic model and the microscopic model in each time step. Thus, the overall macroscopic movement of the runner and the actuators and the microscopic dynamic behavior of the guiding contacts were computed using routines of Matlab ${ }^{\circledR}$ version 2015a. Basic parameters used for numerical investigations were determined and are listed in Table 1. The dimensions of the piezo actuators and therefore their dynamic properties depend on the radius of the hemispheres. Thus, dynamic parameters of the actuators, dependent on the radius, are shown in Table 2 and have been referred from [7]. The preload range and the actuation amplitude range were set according to experimental implementations in [12].

Table 1. Data used for numerical simulations.

\begin{tabular}{cccccc}
\hline $\begin{array}{c}\text { Young's } \\
\text { Modulus (GPa) }\end{array}$ & $\begin{array}{c}\text { Poisson's } \\
\text { Ratio }\end{array}$ & $\begin{array}{c}\text { Friction } \\
\text { Coefficient }\end{array}$ & $\begin{array}{c}\text { Mass of } \\
\text { Runner (g) }\end{array}$ & $\begin{array}{c}\text { Stiffness of } \\
\text { Load Cell (N/m) }\end{array}$ & $\begin{array}{c}\text { Radius of } \\
\text { Hemisphere (mm) }\end{array}$ \\
\hline$E_{1}=370$ (ruby) & $v_{1}=0.33$ & $\mu=0.2 ; 0.3$ & $m_{r}=3$ & $2.5 \times 10^{4}$ & $R=0.25,1$ \\
$E_{2}=210$ (steel) & $v_{2}=0.33$ & - & - & - & - \\
\hline
\end{tabular}

Table 2. Parameter variation dependent on the radius of hemispheres used in simulations.

\begin{tabular}{cccccc}
\hline & $\begin{array}{c}\text { Stiffness of } \\
\text { Actuator (N/m) }\end{array}$ & $\begin{array}{c}\text { Viscosity } \\
\text { Factor (kg/s) }\end{array}$ & $\begin{array}{c}\text { Mass of } \\
\text { Actuator (g) }\end{array}$ & $\begin{array}{c}\text { Preload Range with } \\
\text { Step 0.1 N (N) }\end{array}$ & $\begin{array}{c}\text { Actuation Amplitude } \\
\text { Range (nm) }\end{array}$ \\
\hline$R=0.25$ & $k_{p}=4 \times 10^{6}$ & $c_{p}=10$ & $m_{a}=1.1 \times 10^{-5}$ & $F_{p}=0.1-0.5$ & $0-96$ \\
$R=1$ & $k_{p}=6.5 \times 10^{6}$ & $c_{p}=16.3$ & $m_{a}=2.2 \times 10^{-5}$ & $F_{p}=0.1-1$ & $0-202$ \\
\hline
\end{tabular}

\subsection{Critical Actuation Amplitude}

The model introduced in Section 3 was used to simulate movements of the whole drive with six contact points between the runner and the hemispheres. In fact, as a result of the asymmetric arrangement of the actuators to both sides of the runner, the normal forces applied on the contacts of the two sides were different according to Equation (1). Therefore, the dynamic interaction among these actuators had a strong influence on the critical amplitude. To eliminate the interaction among 
actuators, we first used a one-contact model in which the runner was in contact and was driven by only one actuator. After this, a six-contact model was used for systematical investigations.

\subsubsection{One-Contact Model}

Figure 5a shows the dependency of the step size of the runner on the actuation amplitude with a preload of $0.4 \mathrm{~N}$ and a hemisphere radius of $1 \mathrm{~mm}$. It is seen that when the actuation amplitude is below $50 \mathrm{~nm}$, the step size is equal to zero. This level of actuation amplitude is called the critical amplitude, as stated in the introduction section. By performing similar dynamic simulations to other levels of the preload, we obtained the dependency of the critical amplitude on the preload, as shown in Figure 5b. There are two cases: (i) the actuator is considered as ideally rigid; (ii) the actuator has dynamic properties as a mass-spring-damper system.

In the case of an ideally rigid actuator, we compare the dynamic simulation result with the maximal tangential displacement $u_{x, \max }$ 一the presliding is determined as follows [17,18]:

$$
u_{x, \max }=\frac{3 \mu}{4 G^{*}}\left(\frac{4 E^{*}}{3}\right)^{1 / 3} \frac{F_{p}^{2 / 3}}{R^{1 / 3}}
$$

It is clearly detected in the figure that the value of the critical amplitude at each preload level fits well with $u_{x, \max }$ with a very small discrepancy (compare the two green graphs). This leads to a conclusion that, when the dynamic properties of the actuators as well as their interaction are omitted, the critical amplitude phenomenon is exactly caused by the presliding displacement of the contact $\left(A_{c} \approx u_{x, \max }\right)$.

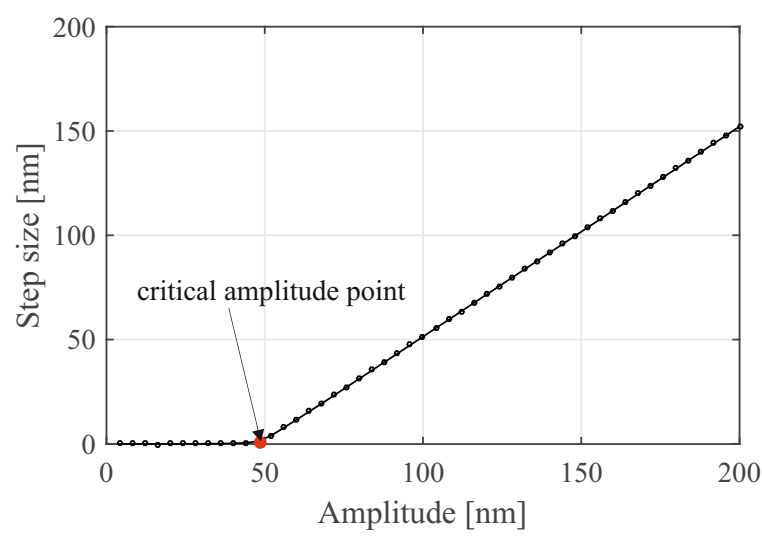

(a)

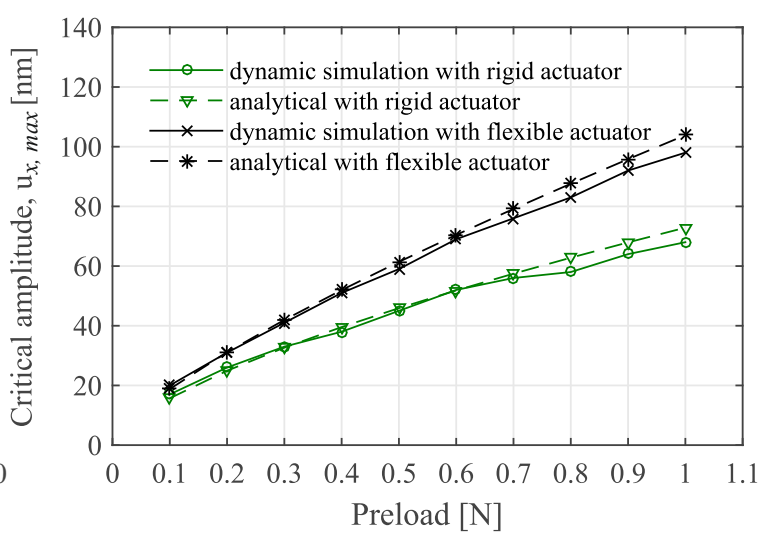

(b)

Figure 5. Simulations of the critical amplitude phenomenon using one-contact model: (a) dependency of the step size on the actuation amplitude; (b) dependency of the critical amplitude on the preload and its relation to $u_{x, \max }$.

In the second case, the dynamic properties of the actuator are considered. The two black graphs in Figure $5 \mathrm{~b}$ compare the dynamic simulation result with the analytical calculation:

$$
A_{c}=u_{x, \max }+u_{x, a}
$$

where $u_{x, a}$ is the tangential deformation of the actuator caused by the friction force. As presented by van der Wulp [5], this parameter is approximately determined by

$$
u_{x, a}=\mu \times \frac{F_{p}}{k_{p}} \times \frac{m_{r}+m_{a}}{m_{r}}
$$


Additionally, the dynamic simulation result agrees excellently with the analytical calculation. This leads to the confirmation that the critical amplitude depends on both the presliding and the dynamic properties of the actuator.

\subsubsection{Six-Contact Model}

Simulations using the six-contact model are shown in Figure 6. On the basis of these results, the interactions among the actuators as well as influencing parameters and critical amplitude are analyzed.

We first consider the case of rigid actuators in Figure 6a. In this figure, the dynamic simulation result of the critical amplitude is compared with the presliding of the contacts arranged to both sides of the runner:

$$
u_{x, \max , j}=\frac{3 \mu}{4 G^{*}}\left(\frac{4 E^{*}}{3}\right)^{1 / 3} \frac{F_{N j}^{2 / 3}}{R^{1 / 3}}(j=1,2)
$$

It is seen that the critical amplitudes do not match the presliding $u_{x, \max , 1}$ or $u_{x, \max , 2}$. In [13] (pp. 53-57), Teidelt used the simplest possible MDR model, allowing for a better understanding of the runner's overall dynamics. She introduced an analytical approximation and suggested that the critical amplitude is equal to

$$
u_{x, \max , e}=\frac{2 u_{x, \max , 1} u_{x, \max , 2}}{u_{x, \max , 1}+u_{x, \max , 2}}
$$

The red graph in Figure 6a shows a fine agreement of $u_{x, \max , e}$ with the dynamic simulation result of $A_{c}$.

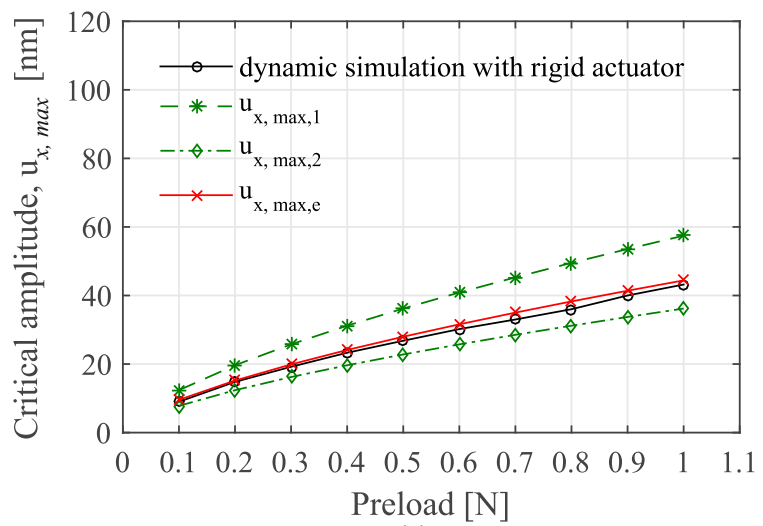

(a)

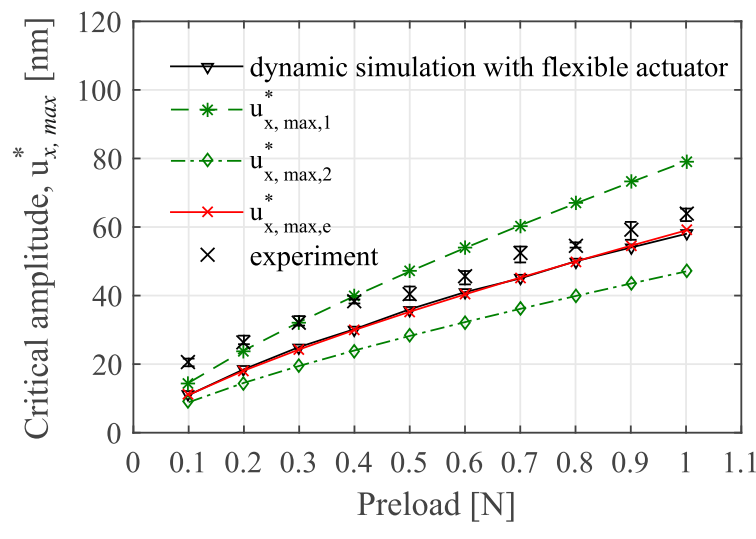

(b)

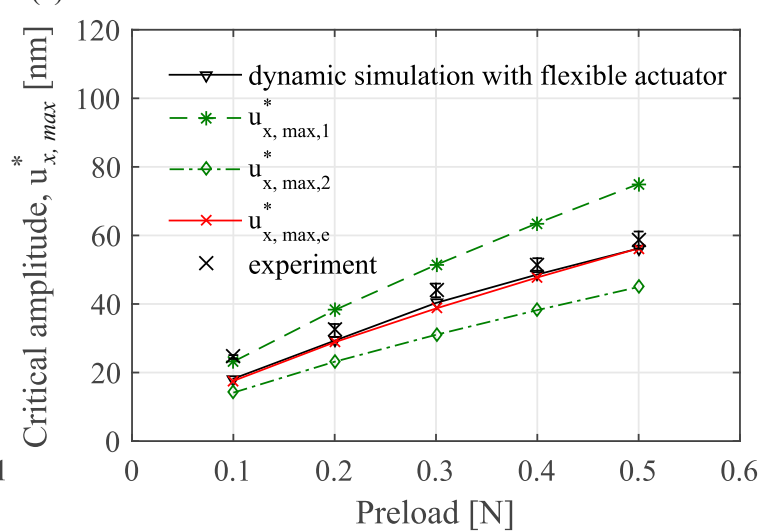

(c)

Figure 6. Simulations of the critical amplitude phenomenon using six-contact model and its relation to $u_{x, \max }$ : (a) ideally rigid actuators with the radius of the hemispheres $R=1 \mathrm{~mm}$; (b) flexible actuators with the radius of the hemispheres $R=1 \mathrm{~mm}$; (c) flexible actuators with the radius of the hemispheres $R=0.25 \mathrm{~mm}$. 
Now we extend the calculation in Equation (11) to the case of flexible actuators by the set of Equations (12)-(14).

$$
u_{x, \max , e}^{*}=\frac{2 u_{x, \max , 1}^{*} u_{x, \max , 2}^{*}}{u_{x, \max , 1}^{*}+u_{x, \max , 2}^{*}}
$$

with

$$
\begin{gathered}
u_{x, \text { max }, j}^{*}=u_{x, \text { max }, j}+u_{x, a, j},(j=1,2) \\
u_{x, a, j}=\mu \times \frac{F_{N j}}{k_{p}} \times \frac{m_{r}+m_{a}}{m_{r}},(j=1,2)
\end{gathered}
$$

In Figure 6b, we compare the dynamic simulation result of $A_{c}$ in the case $R=1 \mathrm{~mm}$ with $u_{x, \max , 1}^{*}$, $u_{x, \max , 2}^{*}, u_{x, \max , e}^{*}$ and experimental data published in [12]. The error bars show the variation in the experimental data within 10 measurements for each preload. A high agreement among $A_{c}, u_{x, m a x, e}^{*}$ and experiment is detected. This confirms our finding that the critical amplitude depends on the presliding of the guiding contacts, the dynamic properties of the actuators and their interaction. This finding is more general because it is valid also for different radii of the hemispheres. As shown in Figure $6 \mathrm{c}$ for the case $R=0.25 \mathrm{~mm}$, a strong accordance of the considered results is obtained.

\subsection{Force Generation}

The drive used in this investigation can also work as a force generator. When the runner moves to touch the load cell, force is generated and measured. The generated force depends on the friction behavior of the guiding contacts, directly relating to the preload, the radius of the hemispheres, the friction coefficient, the material properties, and the actuation amplitude. Detailed descriptions of the force generation can be seen in $[4,10,12,13]$. In [12], the authors performed simulations for the force generation under assumptions that the actuators are ideally rigid and the friction coefficient $\mu$ equals 0.3. The gained results show substantial agreement, for which the critical amplitude fits well with experiments. However, discrepancies in the level of generated forces are still unsolved.

In this work, we have used the extended model introduced in Section 3 to implement dynamic simulations of the force generation. Parameters, representing dynamic behavior of the actuators, are gathered from Table 2. Furthermore, it was found in the experiment work of Lübke [24] that the friction coefficient is equal to 0.2 . Thus, this value was used for the simulations. Figure 7 shows the results for two cases of the hemisphere radius, in which we compare our new simulation results with the simulation and experiment results published in [12]. In Figure 7a, results with preloads of 0.3 and $0.8 \mathrm{~N}$ for $R=1 \mathrm{~mm}$ have been chosen to be shown, whereas Figure $7 \mathrm{~b}$ shows results with preloads of 0.2 and $0.4 \mathrm{~N}$ for $R=0.25 \mathrm{~mm}$. Results with other preload levels did not show different trends. Therefore, for the sake of brevity, we do not show them here.

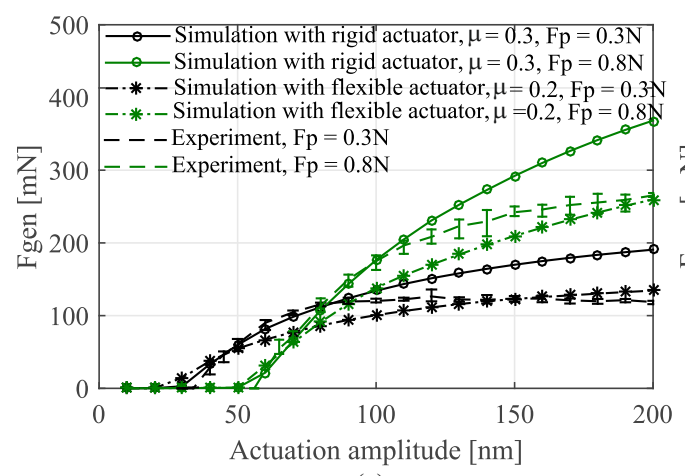

(a)

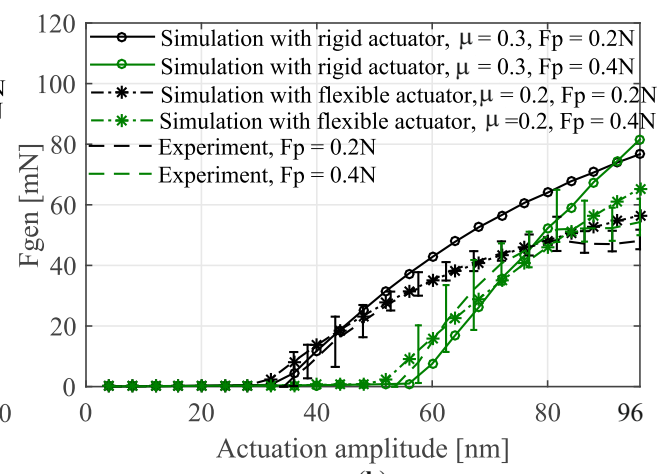

(b)

Figure 7. Dependency of the generated force $F_{g e n}$ on the preload, the friction coefficient, the actuation amplitude and the radius of the hemispheres: (a) $R=1 \mathrm{~mm}$; (b) $R=0.25 \mathrm{~mm}$. 
We can clearly see that the new simulation results (flexible actuator) cover the experiment results well, considering both the critical amplitude and the level of generated forces. Only small discrepancies are detected. On the other hand, the simulation results of the previous work [12] (rigid actuator) can cover only the critical amplitude. Increasing discrepancies in the level of generated forces along with a raising actuation amplitude are recognized. For example, as in Figure 7a, when the actuation amplitude reaches $202 \mathrm{~nm}$ and the preload of $0.8 \mathrm{~N}$, the discrepancy is as large as $115 \mathrm{mN}$.

We can conclude that the friction coefficient and the dynamics of actuators are the causation for the discrepancy in levels of generated forces in [12]. The results of this section again show the higher quality of the extended model.

\section{Waveform Optimization}

\subsection{Theory}

The standard saw-tooth-like driving signal is often used for stick-slip drives. However, an adaption for the waveform can improve performances for the drives, including a decrease in back-step and micro-vibration and thus an increase in step size, driving the frequency and operating velocity. In this paper, we introduce a novel driving waveform allowing us to heighten the step size and thus the operational velocity.

Figure 8a shows the response of the runner when driving with the standard saw-tooth-like waveform in two stick-slip periods. In the stick-phase of the first period, the runner is moved together with the hemispheres. Then, a slip-phase follows, leading to a back-step of the runner. After this phase, the runner starts to vibrate and another period is continued. If the micro-vibration of the runner is not completely damped within the stick-phase of the following period, it will induce chaos to the system, preventing the drives from controlling with a high frequency and thus high operational velocity. The repeatability of the drives is also influenced.

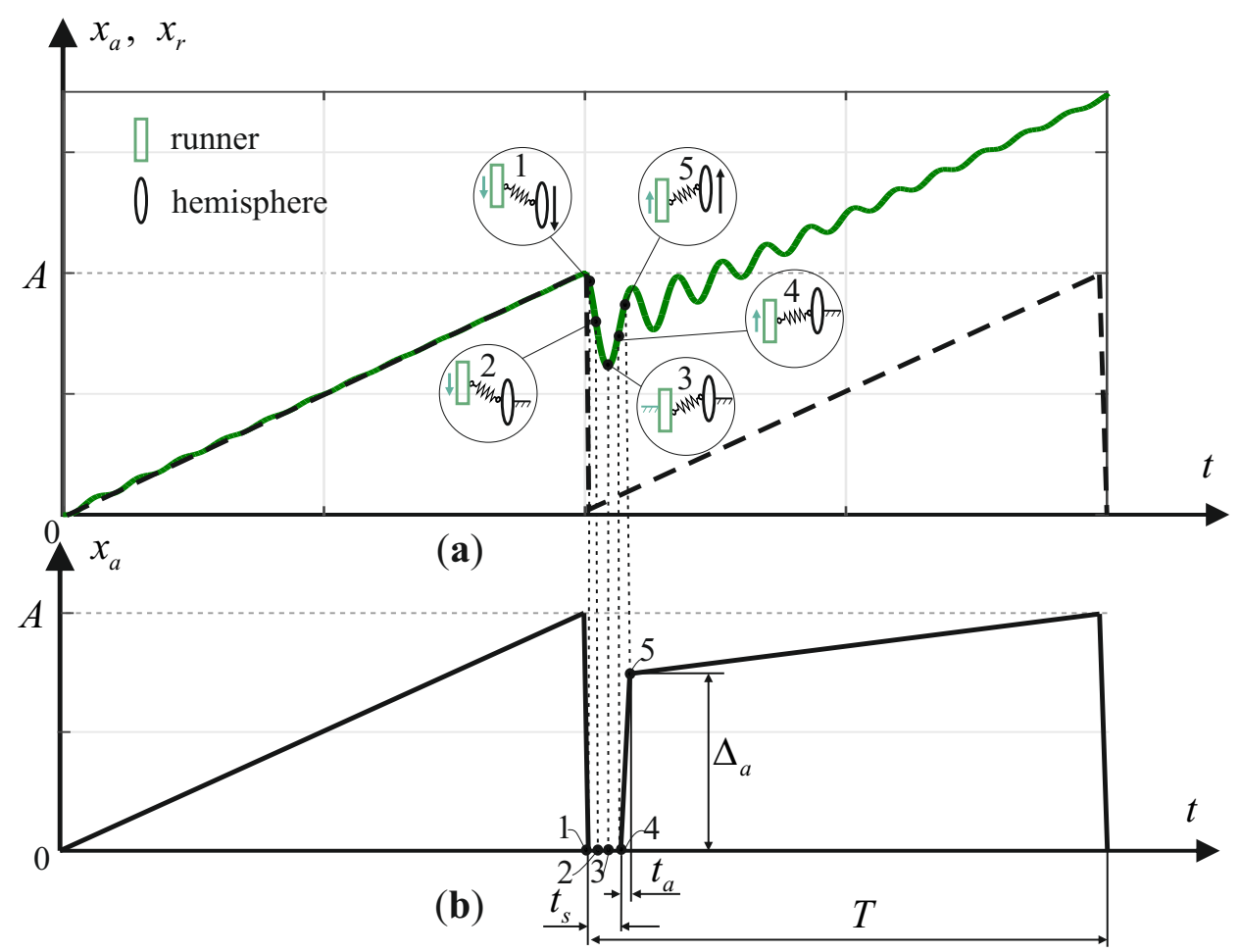

Figure 8. Optimization of the driving waveform: (a) standard saw-tooth-like signal (dashed, black line), response of the runner (continuous, green line) and contact status in circles; (b) optimized waveform. 
In this section, an assumption is made that the piezo actuator is ideally rigid. Thus, the displacement of the hemispheres is linearly proportional to the driving voltage with an amplitude $A$. Each guiding contact is simplified to a spring connecting the runner's surfaces and the hemispheres, as shown in the circles of Figure 8a. Depending on the relative displacement between the runner and the hemispheres, the spring is deformed in the respective direction, showing the contact status. The arrows in the circles express the velocity magnitude and the direction of the displacement of the runner and the hemispheres. If the hemispheres are not moved, they are represented as fixed. The optimized waveform is derived by exploiting the micro-vibration in the transition from slip to stick and analyzing the contacts status. Consequently, the stick-phase is divided into two sub-phases as follows:

- Sub-phase I (settling and acceleration): After the stick-phase of the first period at point 1, the hemispheres are quickly displaced backward to the initial position. Because the runner cannot fully follow the displacement of the hemispheres, it performs a back-step and starts to vibrate. Consequently, the spring is deformed to a maximum, which is equal to the maximal tangential displacement of the contact $u_{x, \max }$. When the slip-phase is finished, instead of moving the hemispheres forward immediately as in the standard saw-tooth-like waveform, we make the hemispheres frozen with a settling time $t_{s}$ (see Figure $8 \mathrm{~b}$ ). The contact status within this time at points 2, 3 and 4 are depicted in the respective circles. At point 2, the runner moves backward, and at point 3, it starts to reverse the movement direction. At point 4 , when the velocity of the runner is maximal, we displace the hemispheres quickly to point 5 with time $t_{a}$ and an amplitude $\Delta_{a}$. Because the runner and the hemispheres are moved in the same direction, the runner is accelerated with the hemispheres. In the acceleration process, if the velocity of the hemispheres $V_{a}=\frac{\Delta_{a}}{t_{a}}$ is too large, a relative slip between the runner and the hemispheres will appear, similar to the slip-phase of the first period. Therefore, $V_{a}$ is chosen to satisfy that it has maximal magnitude and that the runner is still in the stick condition. The stick condition is ensured when $u=\Delta_{a}-u_{r} \leq u_{x, \max }$, where $u$ and $u_{r}$ are the relative displacement between the runner and hemispheres and the displacement of the runner, respectively.

- Sub-phase II (slip): At the end of the acceleration at point 5, the runner has the highest velocity, and thus the kinetic energy and the potential energy due to the deformation of the spring are maximal. We suddenly slow down the hemispheres. As a result, all the energy of the runner makes it quickly slip forward with a displacement that is sustainably larger than the displacement of the hemispheres. This leads to a larger step size for the runner in comparison with that of the standard saw-tooth-like driving waveform.

Parameters for the optimized waveform, $t_{s}, t_{a}$ and $\Delta_{a}$, depend on the dynamic response of the runner and the contact behavior influenced by the normal force $F_{N}$, the radius of the hemispheres $R$, the material properties $\left(G^{*}, E^{*}\right.$ and $\left.v\right)$ and the friction coefficient $\mu$. By analyzing the movement of the runner, we can determine the frequency of the micro-vibration $f_{s}$ and the velocity $V_{r}$. Applying the above-proposed condition yields

$$
t_{s}=\frac{1}{2 f_{s}} ; u=\Delta_{a}-u_{r}=\left(V_{a}-V_{r}\right) t_{a}=u_{x, \max }
$$

It is noted that the optimized driving signal described above is based on the analysis of the response of the system to the saw-tooth. In fact, the response of the system to the optimized waveform may differ from that of the saw-tooth. However, we strongly believe that the difference is very slight. Therefore, our method is still valid. In the following section, the validation of the method is shown.

\subsection{Results}

The efficiency of the new waveform is evaluated by comparing simulation results. Figure 9a shows the simulation results of the step size of the runner with the standard saw-tooth waveform and the optimized waveform. Simulations of three periods were performed with a preload of $0.4 \mathrm{~N}$, 
a radius of the hemispheres of $1 \mathrm{~mm}$, an actuation amplitude of $202 \mathrm{~nm}$ and a driving frequency of $100 \mathrm{~Hz}$. Parameters for the optimized waveform were calculated from Equation (15). As seen in this figure, the step size of the runner, when the optimized waveform was used, was significantly larger than that of the standard saw-tooth waveform. After three periods, the optimized waveform drove the runner to a position of $716 \mathrm{~nm}$, while only $552 \mathrm{~nm}$ was obtained with the standard waveform. Because the optimization was started after the first period, the step size was calculated to increase by about $46.8 \%$, leading to higher operational velocity for the runner.

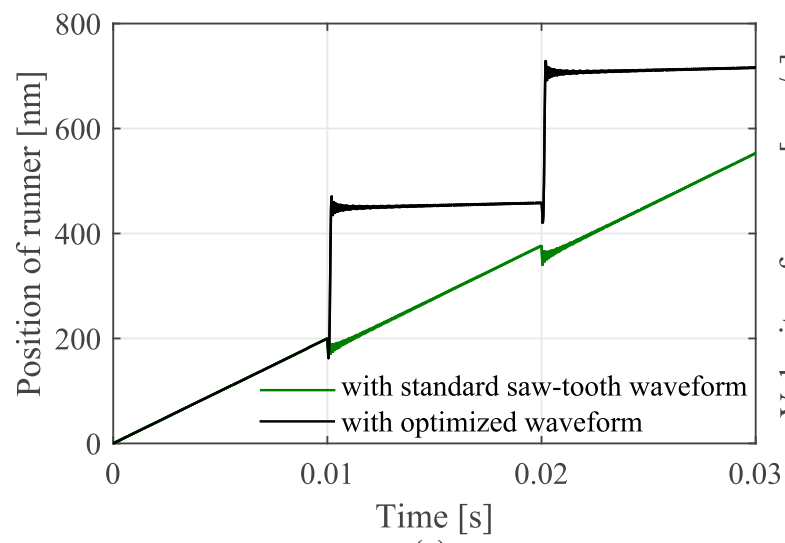

(a)

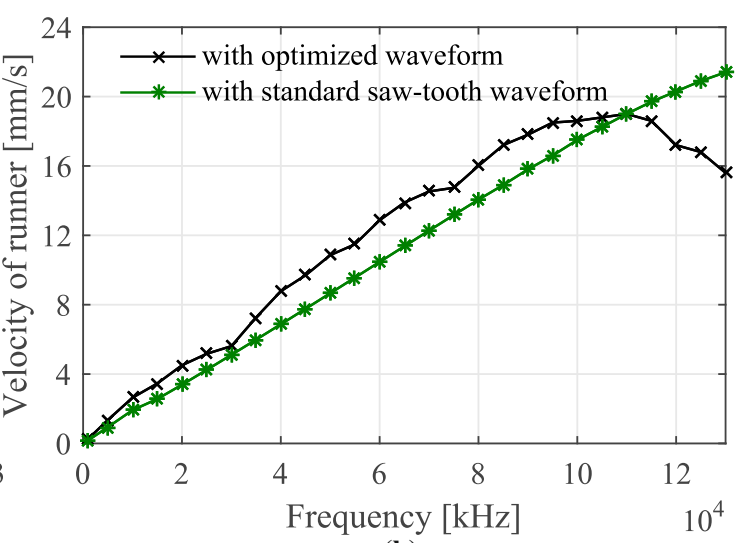

(b)

Figure 9. Comparison of simulation results with standard saw-tooth waveform and optimized waveform: (a) step size at driving frequency $f=100 \mathrm{~Hz}$; (b) dependency of the average velocity of the runner on driving frequencies.

The average velocity of the runner depends on both the step size and driving frequency; it is a multiple of the driving frequency and step size. In fact, the higher the driving frequency, the more chaos that is induced to the system, leading to decreases in the step size. Thus, it is of importance to investigate the influences of the driving frequency on the operational velocity. The dependency of the average velocity of the runner on the driving frequency is shown in Figure $9 b$. Parameters for simulations were set similar to the case of Figure 9a. The frequency was varied in a range from 1 to $130 \mathrm{kHz}$ with a step of $5 \mathrm{kHz}$. It is clear that when the driving frequency was below $110 \mathrm{kHz}$, the velocity of the runner with the optimized waveform was larger than that of the standard waveform. The maximal velocity increment was about $2.5 \mathrm{~mm} / \mathrm{s}$. This increment is substantially significant for the micro-drive. It is also detected that when the driving frequency was over $110 \mathrm{kHz}$, the velocity of the runner with the optimized waveform began decreasing, while the velocity with the standard waveform still increased. This is explained by the very high frequencies making the period too short, not providing enough time for settling. However, practical evidence shows that it is impossible to control the drives with frequencies above $110 \mathrm{kHz}$, as chaos of the system makes the runner unstable. Thus, with frequencies under $110 \mathrm{kHz}$, the optimized waveform can be used efficiently.

The optimized waveform is further characterized by investigating the influence of the preload, the radius of the hemispheres and Young's modulus of the runner on the operational velocity, as shown in Figure 10. When these influencing parameters were varied, the input parameters for the optimized waveform were respectively changed. Consequently, the average velocity of the runner was influenced. In the following section, these influences are described.

A suitable choice of the preload level can help to avoid wear for the guiding contacts as well as maximize the velocity for the drive. Figure 10a shows the dependency of the reachable maximal velocity of the runner on the preload at maximal controllable frequencies. It is observed that a reachable maximal velocity of $19 \mathrm{~mm} / \mathrm{s}$ is achieved at the preload level of $0.4 \mathrm{~N}$ and driving frequency of $110 \mathrm{kHz}$. With higher preloads, for example $1 \mathrm{~N}$, the velocity is reduced because the back-step becomes larger. For a smaller preload, such as $0.1 \mathrm{~N}$, the back-step becomes smaller. However, the stick condition 
in the acceleration process cannot be satisfied when the driving frequency is above $80 \mathrm{kHz}$. Thus, the reachable maximal velocity is slightly reduced.

Figure 10b shows the simulation results with two cases of the hemispheres radius. Input parameters have been set similar to the case of Figure 10a. It is clear that the simulations with a hemisphere radius of $1 \mathrm{~mm}$ generate a higher velocity for the runner in comparison to the case with a radius of $0.25 \mathrm{~mm}$. With the larger radius, the contact stiffness is stiffer and the distribution of the normal pressure in the contact is more balanced. This results in a higher maximal controllable frequency and a smaller back-step. Consequently, the velocity increases.

The influence of the runner's material properties on the velocity with the optimized waveform is shown in Figure 10c. At a preload of $0.4 \mathrm{~N}$ and an actuation amplitude of $202 \mathrm{~nm}$, simulations with steel $\left(E_{2}=210 \mathrm{MPa}\right)$ and molybdenum $\left(E_{2}=310 \mathrm{MPa}\right)$ were performed. The results show that the influence of Young's modulus of the runner can be negligible. The reachable maximal velocity of the two cases has only a small discrepancy.

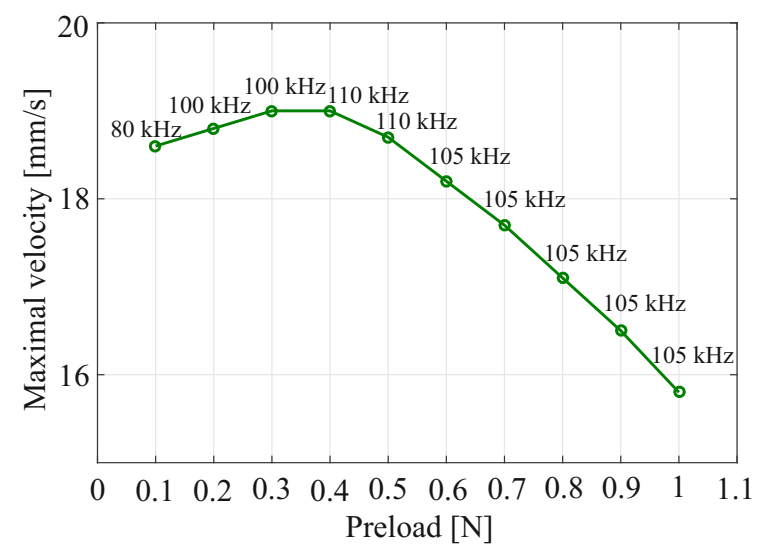

(a)

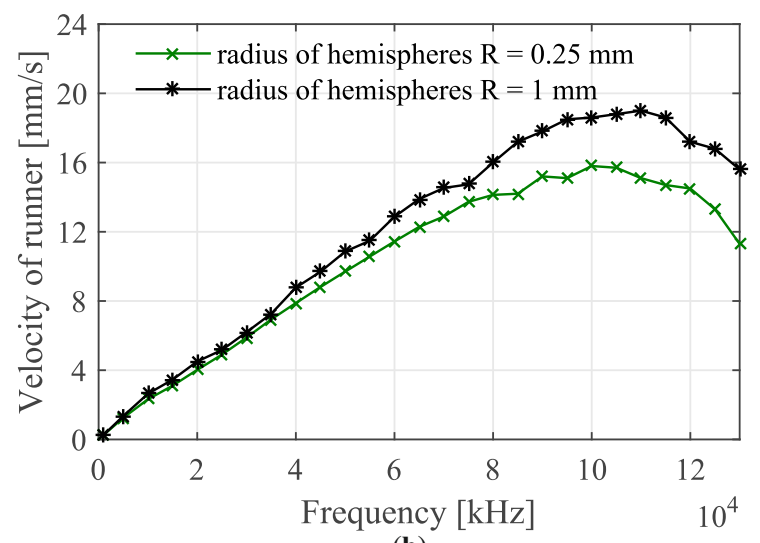

(b)

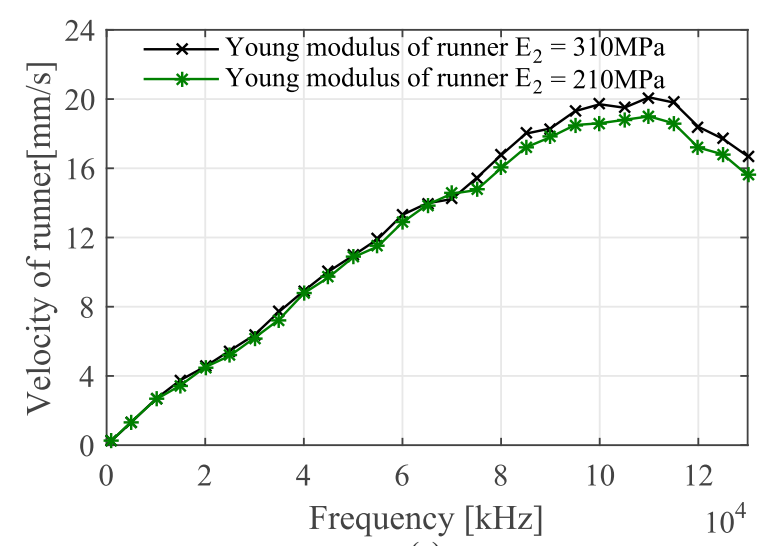

(c)

Figure 10. Influence of important parameters on velocity of the runner: (a) influence of the preload; (b) influence of the radius of the hemispheres; (c) influence of Young's modulus of the runner's material (simulation results).

\section{Conclusions and Outlook}

In this paper, the modeling and the waveform optimization of stick-slip micro-drives have theoretically been studied. We have introduced an extended model for the drive, which can be used to investigate both the macroscopic behavior, including the dynamics of the actuators and the runner, and the microscopic properties of the frictional contacts. Two important characteristics of the drive, consisting of the critical amplitude phenomenon and the force generation, have systematically been analyzed. The achieved results confirm that the critical amplitude depends on the presliding of 
the guiding contacts, the dynamic properties of the actuators and the interaction among them. A more general determination for the critical amplitude is derived. The causation for the discrepancy in levels of the generated forces between the experimental and simulation results of the previously published work has been presented. The use of a more-realistic friction coefficient and the combination of the dynamics of the actuators and the runner in our introduced model allow for much better results. The simulation of the force generation fits excellently with the experiment.

We have introduced a novel driving waveform for the investigated drive that is based on exploiting the micro-vibration and considering the contact status. The optimized waveform is received under an assumption that the actuators are ideally rigid. This waveform allows for a maximal $15 \%$ higher operational velocity for the drive in comparison with the standard saw-tooth waveform. Influences of parameters such as the preload, the radius of hemispheres and Young's modulus on the runner's velocity have been investigated. The preload and the radius of the hemispheres have been found to have significant influences, while the influence of Young's modulus can be negligible.

It is noted that the results obtained in Section 5 are only from the simulations. Therefore, for future works, we will study influences of the actuator dynamics on the efficiency of the new waveform. Moreover, experimental validation for this waveform is of importance and should therefore be given.

Acknowledgments: This research is funded by the Vietnam National Foundation for Science and Technology Development (NAFOSTED) under Grant No. 107.01-2015.18.

Author Contributions: Xuan-Ha Nguyen proposed the idea for modeling, analyzed the results and wrote the manuscript. Tien-Hiep Mau performed the simulations and gave discussions. Ingo Meyer proposed the idea for the driving waveform and discussed the results with Xuan-Ha Nguyen. Bao-Lam Dang and Hong-Phuc Pham advised the technical content and discussed the results. All authors reviewed the manuscript.

Conflicts of Interest: The authors declare no conflict of interest.

\section{References}

1. Eichhorn, V. Nanorobotic Handling and Characterization of Carbon Nanotubes inside the Scanning Electron Microscope. Ph.D. Thesis, Verlag Dr. Hut, München, Germany, 2011.

2. Zhang, Z.M.; An, Q.; Li, J.W.; Zhang, W.J. Piezoelectric frictional inertia actuator: A critical review and future perspective. Int. J. Adv. Manuf. Technol. 2012, 62, 669-685.

3. Hunstig, M. Piezoelectric inertia motors-A critical review of history, concepts, design, applications, and perspectives. Actuators 2017, 6, 7, doi:10.3390/act6010007.

4. Nguyen, X.H. Simulation, Validation and Optimization of Stick-Slip Drives for Nanorobotic Applications. Ph.D. Thesis, The University of Oldenburg, Oldenburg, Germany, 2014; pp. 13-16.

5. Van der Wulp, H. Piezo-Driven Stages for Nanopositioning with Extreme Stability: Theoretical Aspects and Practical Design Considerations. Ph.D. Thesis, Delft University Press, Delft, The Netherlands, 1997.

6. Hunstig, M.; Hemsel, T.; Sextro, W. Modelling the friction contact in an inertia motor. J. Intell. Mater. Syst. Struct. 2013, 24, 1380-1391.

7. Breguet, J.M. Stick and Slip Actuators. Ph.D. Thesis, Ecole Polytechnique Federale De Lausanne (EPFL), Lausanne, Switzerland, 1998.

8. Peng, J.Y.; Chen, X.B. Modeling of piezoelectric-driven stick-slip actuators. IEEE/ASME Trans. Mechatron. 2011, 16, 394-399.

9. Edeler, C.; Meyer, I.; Fatikow, S. Modeling of stick-slip micro-drives. J. Micro-Nano Mechatron. 2011, 6, 65-87.

10. Edeler, C. Modellierung und Validierung der Krafterzeugung mit Stick-Slip-Antrieben fuer nanorobotische Anwendungen. Ph.D. Thesis, Verlag Dr. Hut, München, Germany, 2011.

11. Teidelt, E.; Willert, E.; Filippov, A.E.; Popov, V.L. Modeling of the dynamic contact in stick-slip microdrives using the method of reduction of dimensionality. J. Phys. Mesomech. 2012, 15, 287-292.

12. Nguyen, H.X.; Teidelt, E.; Popov, V.L.; Fatikow, S. Modeling and waveform optimization of stick-slip microdrives using the method of dimensionality reduction. J. Arch. Appl. Mech. 2016, 86, 1771-1785.

13. Teidelt, E. Friction Induced Motion and Control of Friction. Ph.D. Thesis, Berlin University of Technology, Berlin, Germany, 2014. 
14. De Canudas, W.; Olsson, H.; Astrom, K.J.; Lischinsky, P. A new model for control of systems with friction. IEEE Trans. Autom. Control 1995, 40, 419-425.

15. Dupont, P.; Hayward, V.; Armstrong, B.; Altpeter, F. Single state elastoplastic friction models. IEEE Trans. Autom. Control 2002, 47, 787-792.

16. Dahl, P.R. A Solid Friction Model; Aerospace Corp: El Segundo, CA, USA, 1986.

17. Popov, V.L. Basic ideas and applications of the method of reduction of dimensionality in contact mechanics. J. Phys. Mesomech. 2012, 15, 254-263.

18. Popov, V.L. Method of reduction of dimensionality in contact and friction mechanics: A linkage between micro and macro scales. Friction 2013, 1, 41-62.

19. S̆piller, M.; Hurák, Z. Hybrid charge control for stick-slip piezoelectric actuators. J. Mechatron. 2011, 21, 100-108.

20. Bergander, A.; Breguet, J.M. Performance improvements for stick-slip positioners. Proc. Int. Symp. Micromechatron. Hum. Sci. 2003, doi:10.1109/MHS.2003.1249910.

21. Hunstig, M.; Hemsel, T.; Sextro, W. Stick-slip and slip-slip operation of piezoelectric inertia drives-Part I: Idea excitation. Sens. Actuators A Phys. 2013, 200, 90-100.

22. Hunstig, M.; Hemsel, T.; Sextro, W. High-velocity operation of piezoelectric inertia motors: Experimental validation. Arch. Appl. Mech. 2016, 86, 1733-1741.

23. Teidelt, E.; Nguyen, X.H.; Fatikow, S.; Popov, V.L. Numerische simulation von nano-stick-slip positionierungsantrieben mittels der methode der dimensionsreduktion. In Proceedings of the TribologieFachtagung, Göttingen, Germany, 30 September-2 October 2013.

24. Lübke, M. Aufbau Eines Reibkraftmessstandes und Durchführung von Messreihen; Universität Oldenburg: Oldenburg, Germany, 2010. (In German)

(C) 2018 by the authors. Licensee MDPI, Basel, Switzerland. This article is an open access article distributed under the terms and conditions of the Creative Commons Attribution (CC BY) license (http:/ / creativecommons.org/licenses/by/4.0/). 